\title{
A Doppler aliasing free micro-motion parameter estimation method in the terahertz band
}

\author{
Qi Yang, Bin Deng, Hongqiang Wang and Yuliang Qin*
}

\begin{abstract}
Micro-Doppler, induced by micro-motion of targets, is an important characteristic for target recognition once extracted via parameter estimation. However, micro-Doppler is usually too significant to result in aliasing in the terahertz band. According to this problem, a Doppler aliasing free micro-motion parameter estimation method based on the modulo Generalized Hough transform is proposed in this paper. Its basic idea is to search and match the parameters of aliasing micro-Doppler curves in the time-frequency distribution image. The high estimation precision and excellent noise immunity of this method are verified through simulations based on CST data and experiments based on a $0.33 \mathrm{THz}$ radar system.
\end{abstract}

Keywords: Terahertz radar, Micro-Doppler, Generalized Hough Transform, Doppler aliasing, Parameter estimation

\section{Introduction}

Terahertz $(\mathrm{THz})$ waves usually refer to electromagnetic waves with frequencies between 0.1 and $10 \mathrm{THz}$. The terahertz band lies between the millimeter wave and infrared, which is a transitional band from electronics to photonics. Its position in the spectrum confers special properties and applications on terahertz waves that differ from other bands [1-3]. With breakthroughs in terahertz sources, signal detectors and other devices, the terahertz radar technology has developed rapidly, and many terahertz radar systems have been established, mainly for the study of high resolution imaging [4-7]. Some terahertz devices and radar systems are getting mature, and examples are the $0.85 \mathrm{THz}$ vacuum-based power amplifier designed by Northrop Grumman Corporation [8] and the ultra-high-resolution radar Miranda 300 designed by the FGAN Research Institute for High Frequency Physics and Radar Techniques [9]. However, researches on micro-motion targets, as a common type of object in the real world, are still very limited, although terahertz waves, due to their short wavelengths, are more sensitive to Doppler than microwaves and hence more suited for micro-Doppler imaging. Also, the

\footnotetext{
* Correspondence: 424206134@qq.com

College of Electronic Science and Engineering, National University of Defense Technology, Changsha 410073, China
}

serious atmospheric attenuation of terahertz waves has little influence on their applications in exoatmosphere or near space. As a result, the research on parameter estimation of micro-motion targets with terahertz radar is significant. Despite the advantages mentioned above, micro-motion parameter estimation in the terahertz band faces a significant hurdle, i.e., the Doppler aliasing induced by the inadequate pulse repetition frequency (PRF). Terahertz radar usually utilizes frequency modulated continuous wave (FMCW) signals due to low peak power requirement. For example, both the $220 \mathrm{GHz}$ COBRA radar of FGAN [6] and the $580 \mathrm{GHz}$ imaging radar of Jet Propulsion Laboratory (JPL) [7] utilize FMCW. In order to ensure the linearity of the FMCW transmitted signal, the equivalent PRF can't be too large, and therefore the limited PRF sets an upper limit to the maximal observable non-aliasing Doppler values. Specifically, the Doppler frequency can be unambiguously observed only when it lies in the interval between $-\mathrm{PRF} / 2$ and PRF/2, and it will be aliasing or folded up when Doppler values are outside this interval. In addition, micro-Doppler in the terahertz band tends towards being aliasing as the carrier frequency is much higher than that in the microwave band.

For micro-motion in the terahertz band, Robert W. McMillan, et al. introduced an experimental coherent 


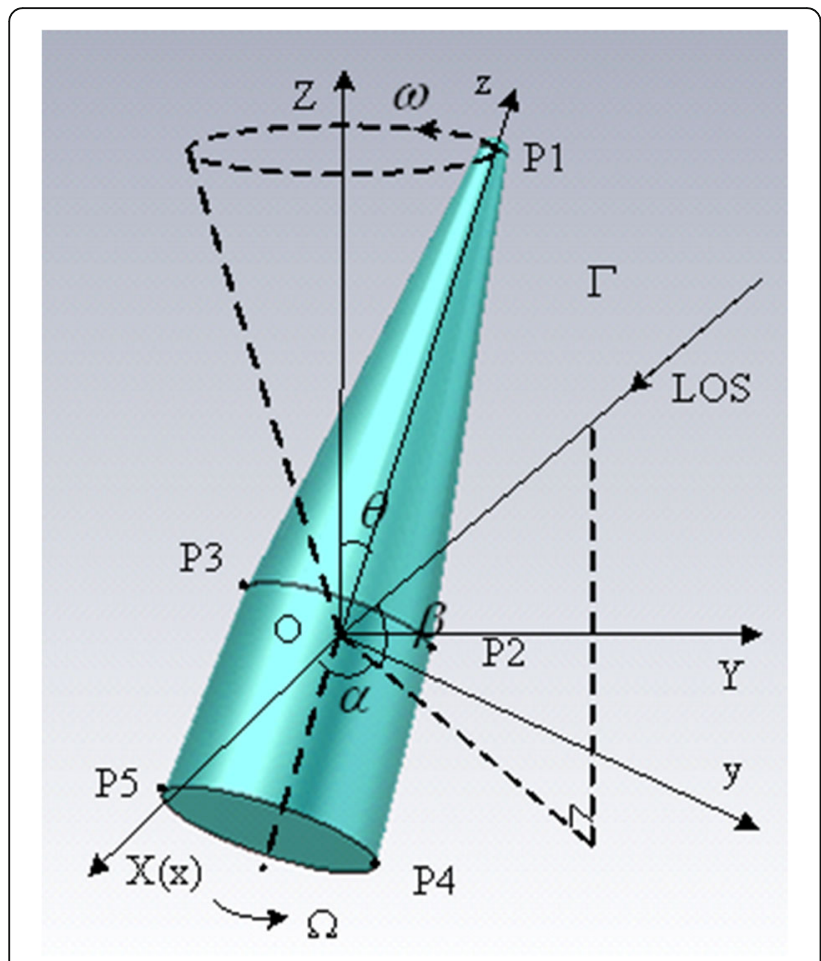

Fig. 1 The diagram of a precession model in CST

pulsed radar operating at $225 \mathrm{GHz}$ early in 1991, and obtained the Doppler spectrum of a stationary truck with its engine running [10]. Li J et al. performed a theoretical analysis of typical micro-motion forms to establish the micro-Doppler signature model, and then compared the micro-Doppler effect in the terahertz band and that in $\mathrm{X}$ band by using the joint time-frequency analysis method [11]. X. Zhengwu et al. analyzed the characteristics of micro-motion and investigated methods for motion parameter estimation and micro-Doppler signature extraction by the Radon transform [12]. However, the parameter estimation method in micro-Doppler aliasing situation was not considered, just because the micro-Doppler values they assumed are relatively smaller than $\mathrm{PRF} / 2$. In practice, micro-
Doppler aliasing in the terahertz radar system is inevitable, and thus these algorithms are inapplicable for our concerns.

Herein, we propose an algorithm for micro-motion parameter estimation based on the modulo Generalized Hough transform (GHT). Its main idea is derived from the GHT in image processing field, which searches sinusoidal curves or other types of curves hidden in images. However, the GHT cannot be used in the aliasing situation directly. In order to obtain micro-motion parameters in the aliasing situation, we firstly obtain the time-frequency distributions of micro-motion targets, and then match the micro-Doppler curves of scattering centers in the time-frequency image with aliasing reference curves obtained by modulo. Finally, we map the parameters of curves to parameter spaces and estimate them by extracting positions of peaks in parameter spaces.

The paper is organized as follows. Section 2 analyzes the micro-Doppler characteristics of a precession warhead model in the terahertz band. In section 3, an aliasing free parameter estimation method based on modulo GHT is proposed and the detailed procedures are given. In section 4 , the simulation and experimental results are shown and the performance of the method is analyzed. The conclusions are presented in section 5.

\section{Micro-Doppler characteristics of a precession warhead model}

2.1 Theoretical model of echo signals

The intended target in this paper is a precession rotational symmetric warhead model, which is a simplified model of the ballistic missile target during the midcourse. The simulation data of this paper come from a $3 \mathrm{D}$ electromagnetic simulation software named computer simulation technology (CST). The diagram of the warhead model observed by radar is shown in Fig. 1.

A reference frame $\mathrm{O}-\mathrm{XYZ}$ that takes the mess center of the model as the origin is established. Considering the model precessing around $\mathrm{O}-\mathrm{Z}$ axis, the spin angular velocity is $\Omega$, the precession angular velocity is $\omega$, the precession
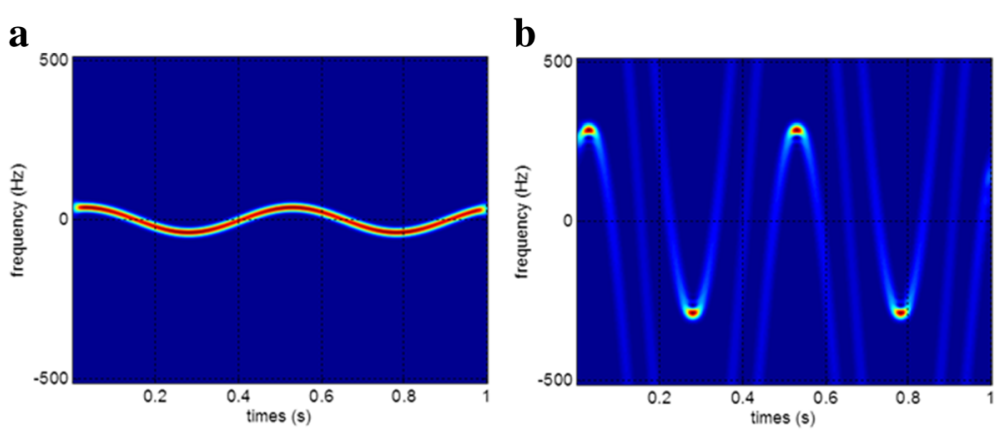

Fig. 2 Time-frequency distributions of a micro-motion scattering center. a The non-aliasing situation at $10 \mathrm{GHz}$. b The aliasing situation at $0.33 \mathrm{THz}$ 

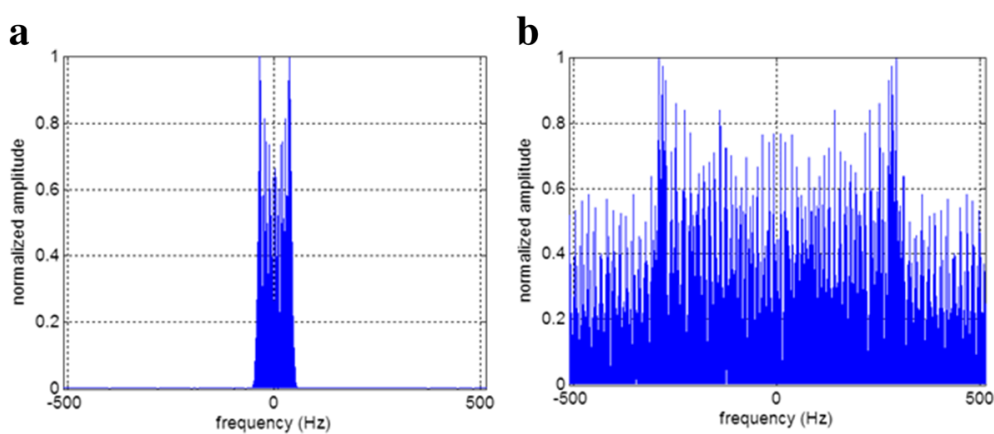

Fig. 3 Fourier transform results of echo signals. a The non-aliasing situation at $10 \mathrm{GHz}$. b The aliasing situation at $0.33 \mathrm{THz}$

angle is $\theta$, the azimuth and pitch angles of the light of sight (LOS) are $\alpha$ and $\beta$, the initial distance between the radar and the model is $R_{0}$. According to the theoretical calculation and the experimental measurement, every scattering center corresponds to a discontinuity in the Stratton-Chu integral, that is the discontinuities of the curvature or surface from the view point of geometry. For the model shown in Fig. 1, the scattering centers include the cone-top P1, the discontinuities in the intersection line of the plane $\Gamma$ (plane $\Gamma$ is composed of the LOS and the symmetrical axis of the model $\mathrm{Oz}$ ) and the model P2, P3, P4, and P5.

Because the model is rotational symmetric, spin makes no difference to the echo modulation. Thus, the radial distance $r(t)$ between the radar and any scattering center located at $(x, y, z)$ on the target may be written as:

$$
\begin{aligned}
r(t)=R_{0} & +\sin \beta(y \sin \theta+z \cos \theta) \\
& +(x \cos \alpha+y \sin \alpha \cos \theta-z \sin \alpha \sin \theta) \cos \beta \cdot \cos \omega t \\
& +(x \sin \alpha-y \cos \alpha \cos \theta+z \cos \alpha \sin \theta) \cos \beta \cdot \sin \omega t \\
=R_{0} & +\sin \beta(y \sin \theta+z \cos \theta)+A \cos (\omega t+\varphi)
\end{aligned}
$$

In this equation, $A$ is amplitude modulated coefficient, and $\varphi$ is the initial phase. The micro-Doppler $f_{d}(t)$ of the scattering center with a carrier frequency $f_{0}$ is:

$$
\begin{aligned}
f_{d}(t) & =-\frac{2 f_{0}}{c} \frac{d r(t)}{d t}=\frac{2 f_{0}}{c} A \omega \sin (\omega t+\varphi) \\
& =A_{\omega} \sin \left(\omega t+\varphi_{\omega}\right)
\end{aligned}
$$

where $A_{\omega}$ is the maximal micro-Doppler frequency, and $\varphi_{\omega}$ is its initial phase. The micro-Doppler of scattering centers on the model are sinusoidally modulated with a period $2 \pi / \omega$. Therefore, the extraction of precession parameters is equivalent to the estimation of periods, amplitudes and initial phases of sinusoidal curves in time-frequency images. In general, amplitudes and initial phases usually reflect the relative positions of scattering centers, which can be used for space reconstruction and imaging of micro-motion targets.

\subsection{Characteristics of Doppler aliasing}

There is no sufficient available real data of precession targets in terahertz band to illustrate the characteristics of Doppler aliasing limiting by the current devices and conditions of our laboratory, and consequently the CST data are adopted to simulate the reality. It is obvious that micro-Doppler $f_{d}$ is in proportion to the carrier frequency $f_{0}$ through Eq. (2). The higher the $f_{0}$, the more evident is the micro-Doppler effect. Therefore, micro-Doppler values in terahertz band are far larger than that in microwave band under
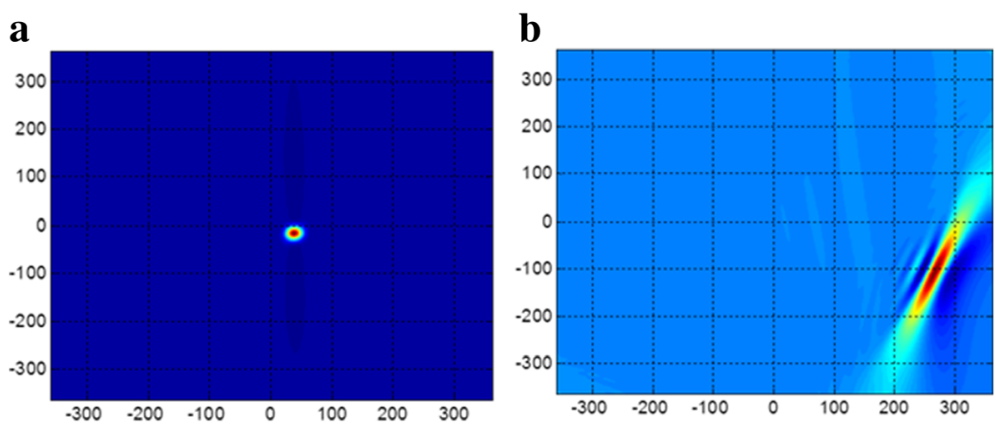

Fig. 4 IRT results of echo signals. a The non-aliasing situation at $10 \mathrm{GHz}$. b The aliasing situation at $0.33 \mathrm{THz}$ 

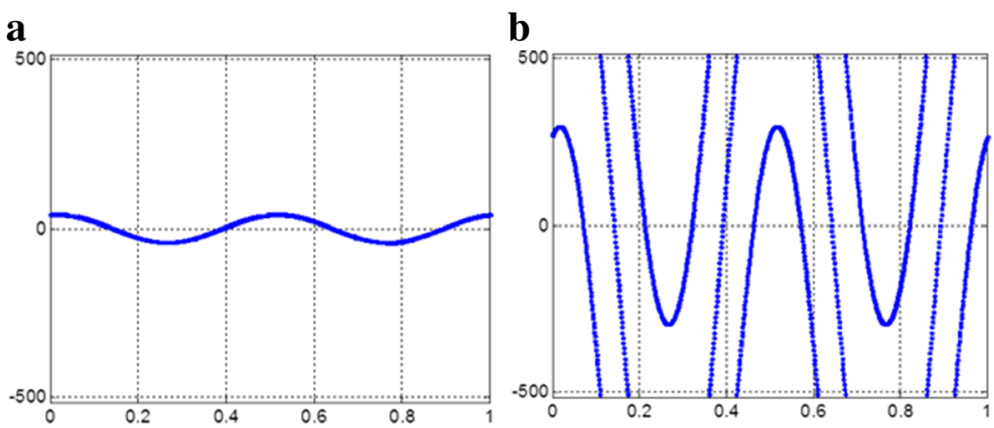

Fig. 5 Matching pixels of micro-Doppler curves. a The non-aliasing situation. b The aliasing situation

identical motion conditions. Suppose the PRF of the transmitted signal is $1 \mathrm{KHz}$, and the maximum micro-Doppler value of a micro-motion scattering center is about $40 \mathrm{~Hz}$ when the carrier frequency is $10 \mathrm{GHz}$ (Fig. 2a), while at $0.33 \mathrm{THz}$ it would reach $1320 \mathrm{~Hz}$, which substantially exceeds the up limit of $\mathrm{PRF} / 2$, hence aliasing is present (Fig. 2b).

In micro-Doppler aliasing situations, the observed Doppler values are no longer the correct values, but are projections on the interval from $-\mathrm{PRF} / 2$ to $\mathrm{PRF} / 2$. Estimation of the real Doppler value for SAR or ISAR becomes necessary as we cannot obtain perfect SAR/ ISAR images without the real Doppler value.

\subsection{Aliasing effect on parameter estimation methods}

Traditional parameter estimation methods such as the Fourier transform and the Inverse Radon transform (IRT) are not applicable for the aliasing situation because aliasing changes the signal properties and destroys the completeness of time-frequency curves.

The spectrum results of echo signals at $10 \mathrm{GHz}$ and $0.33 \mathrm{THz}$ are shown in Fig. 3, respectively. We can easily find that the abscissa values of the peaks in the spectra correspond to the maximal micro-Doppler values in non-aliasing situation. In aliasing situation on the contrary, abscissa values of the peaks are no longer correspond to the maximal micro-Doppler values but the aliasing values, and suggests that the Fourier transform is not suitable for aliasing situations.

The IRT is an effective method for the detection of sinusoidal curves. It can map sinusoidal curves in digital images to peaks in parameter spaces, and then obtain the parameters according to positions of peaks in parameter spaces. However, it is not applicable in microDoppler aliasing situations for the completeness of sinusoidal curves is destroyed. The IRT results of echo signals at $10 \mathrm{GHz}$ and $0.33 \mathrm{THz}$ are shown in Fig. 4, and we cannot obtain any useful information from the IRT result of aliasing micro-Doppler in Fig. $4 \mathrm{~b}$ although the IRT is a powerful tool (see [13]). As a result, we have to study new parameter estimation algorithms for microDoppler aliasing situations.

\section{Aliasing free parameter estimation based on modulo GHT}

\subsection{Algorithm principle}

The algorithm in this paper depends on the timefrequency distributions, and we chose short-time Fourier transform (STFT) as a basis as it has no cross term interference. There is a phase error because of the discrepancy in the start time between STFT and the signal and can be compensated during parameter
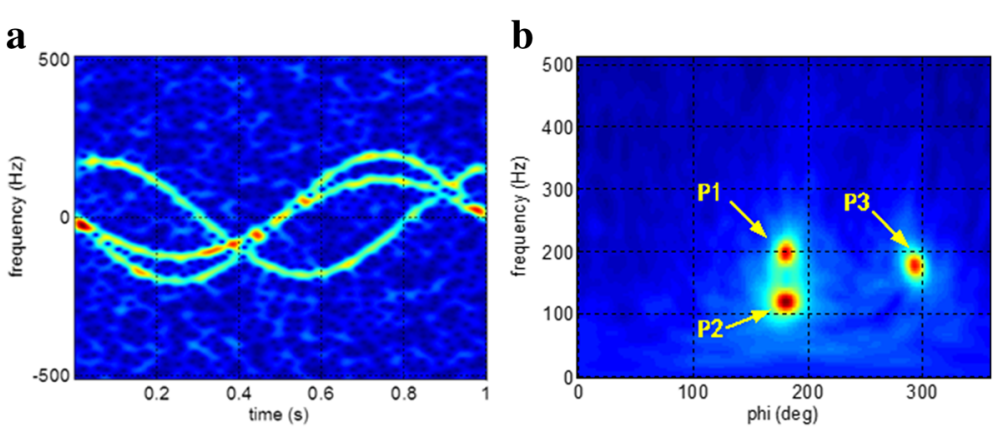

Fig. 6 Results of experiment No. 1. (carrier frequency: 40 GHz, SNR: 3 dB, scattering centers: P1, P2, and P3). a The time-frequency image. b The parameter space image 

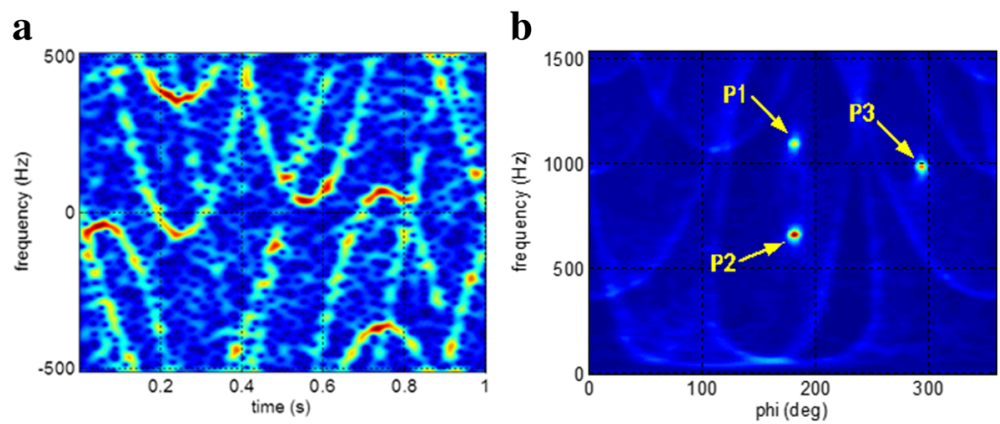

Fig. 7 Results of experiment No. 2. (carrier frequency: 220 GHz, SNR: 3 dB, scattering centers: P1, P2, and P3). a The time-frequency image. b The parameter space image

estimation. We can estimate the micro-motion parameters after obtaining time-frequency distribution images of echo signals. It is well known that GHT is an effective way to detect curves in digital image processing when the curve expressions are known [14-16]. The basic idea of GHT is to map the curves in measurement space to peaks in the parameter space. Curves which share the parameters correspond to the same peak in the parameter space. We can then extract the parameters by identifying the positions of peaks in parameter spaces. Returning to the problem in this paper, the primary objective is to map the aliasing sinusoidal curves in time-frequency distribution images to peaks in parameter spaces. GHT cannot be directly applied to the Doppler aliasing situation as no analytical expressions exist, necessitating an improvement in this case.

We at first establish a parameter space $K=\left(A_{k}, \varphi_{k}, \omega_{k}\right)$ according to the expression of micro-Doppler $f_{d}(t)$. The period of sinusoidal curves is easily obtained by the autocorrelation method in time domain or by the cepstrum method in frequency domain [17], because Doppler aliasing and noise have no effect on the periodicity of signals. Therefore, the parameter space can be reduced to $K=\left(A_{k}, \varphi_{k}\right)$. If there is a sinusoidal curve $f(t)=A_{\omega} \sin \left(\omega t+\varphi_{\omega}\right)$ in a time-frequency image (Fig. 2a), $f(t)$ is similar to the reference curve made up by pixels located at the coordinates $\left(t, A_{k} \sin \left(\omega t+\varphi_{k}\right)\right)$, with just different parameters that are termed as reference pixels. When the target curve and the reference curve have the same parameters, i.e., $A_{k}=A_{\omega}$ and $\varphi_{k}=\varphi_{\omega}$, we call this reference curve the matching curve (Fig. 5a). Their shapes are match well with each other, and the values of matching pixels are larger, the average value of matching pixels is larger, too. If we map the mean of all groups of reference pixels to the parameter space, the mean corresponding to matching curves manifests as peaks in the parameter space. In the aliasing situation, the improvement on GHT is that, if the micro-Doppler is aliasing (Fig. 2b), then the reference curve should be equally aliasing too, i.e. the vertical ordinates of reference pixels modulo PRF (Fig. 5b), so the aliasing microDoppler still matches well with the aliasing reference curve. We then identify the appropriate search scope and step length for the searching algorithm. Finally, we extract positions of peaks and derive the parameters of time-frequency curves. Considering the computation load, we can design a variable step-length searching method. The first step would be to affirm the potential intervals of parameters by choosing a long step length and searching the space parameter. Then, we can estimate parameters accurately in these potential intervals with a short step length. The algorithm proposed in
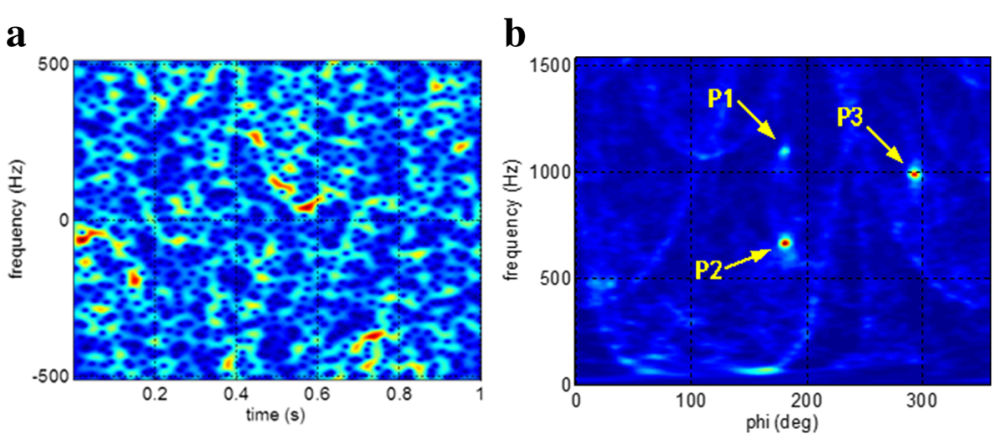

Fig. 8 Results of experiment No. 3. (carrier frequency: $220 \mathrm{GHz}$, SNR: -3 dB, scattering centers: P1, P2, and P3). a The time-frequency image. b The parameter space image 

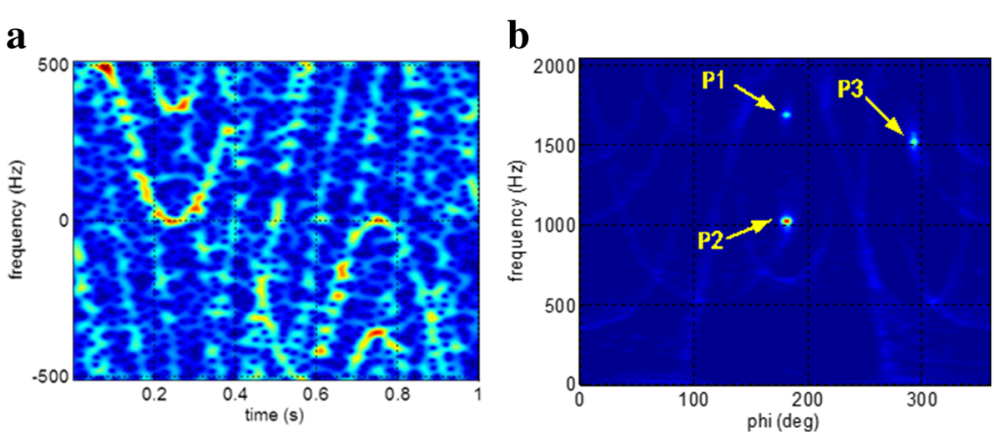

Fig. 9 Results of experiment No. 4. (carrier frequency: 340 GHz, SNR: 3 dB, scattering centers: P1, P2, and P3). a The time-frequency image. b The parameter space image

this paper is applicable in both situations, without prejudging whether aliasing is present or not. Thus, the complexity of this algorithm has been greatly reduced. We call this algorithm the modulo GHT method.

\subsection{Algorithm steps}

To sum up, the parameter estimation method will be carried out in six steps:

(1) Obtain the time-frequency distribution image TF of a micro-motion target by an appropriate timefrequency analysis method (such as STFT).

(2) Estimate the period of micro-motion by the autocorrelation method in order to reduce the parameter space.

(3) Establish a parameter space $K=\left(A_{k}, \varphi_{k}\right)$ and identify an appropriate search scope and step length based on both effectiveness and estimation precision. In this paper, we set the upper limit of the microDoppler frequency $F$ reasonably according to the experimental conditions. The integration time is $T=1 s$, the micro-Doppler step length is $\Delta f=1 \mathrm{~Hz}$, and the phase step length $\Delta \varphi=1^{\circ}$.

(4) Identify the coordinates of reference pixels in the time-frequency distribution image TF, especially in the micro-Doppler aliasing situations. The reference pixel coordinates can be expressed as $\left(t, \bmod \left(A_{k} \sin \left(\omega t+\varphi_{k}\right)+P R F / 2, P R F\right)\right)$, where $\bmod (\cdot)$ is the modulo operation and that is different from GHT.

(5) Search and match the parameters, and average the values of reference pixels in each group in order to reduce detrimental effects of noise.

(6) Extract positions of peaks in the parameter space. Their abscissa values represent the initial phases and vertical ordinate values of the maximum microDoppler values.

\section{Data processing results and analysis}

\subsection{Simulation results}

We design a group of simulation experiments to validate our algorithm above. Simulation results of each experiment are shown in Figs. 6, 7, 8, 9, and 10. The relative frequency and phase errors of each experiment are shown in Fig. 11.

For each group of results, (a) is the time-frequency image of scattering centers, and (b) is the parameter space obtained through the modulo GHT method. The peaks in parameter spaces correspond to time-frequency curves of scattering centers. The results taken together

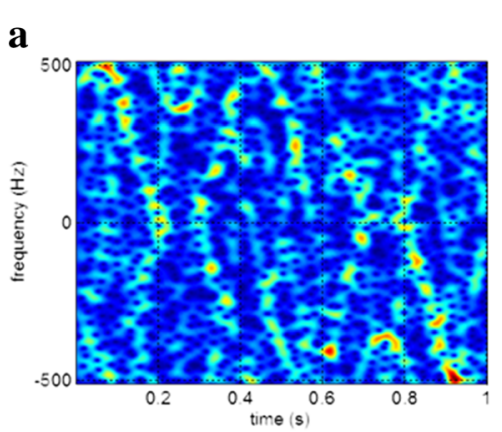

b

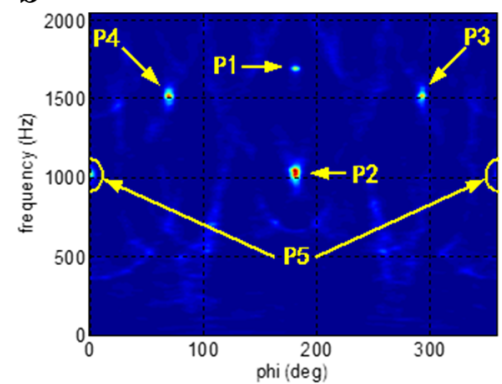

Fig. 10 Results of experiment No. 5. (carrier frequency: 340 GHz, SNR: 3 dB, scattering centers: P1, P2, P3, P4, and P5). a The time-frequency image. b The parameter space image 


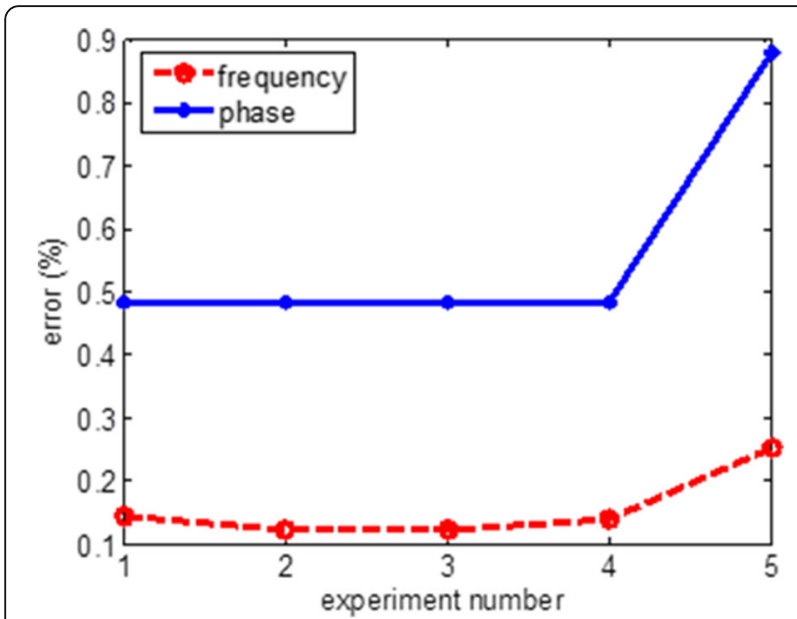

Fig. 11 The relative errors

show that the algorithm based on modulo GHT does well both in situations of aliasing and non-aliasing, and the noise immunity is very good from the results of No. 2 and No. 3. We can estimate the parameters even though the shapes of time-frequency distributions are no longer observed due to low SNR, i.e., we can still find the right peaks in the parameter space when the SNR is $-3 \mathrm{~dB}$, even though the timefrequency distribution image is polluted severely by noise. Furthermore, the algorithm has a high reliability in multi-scattering centers situations from the comparison of No. 5 with others. Finally, the relative frequency and phase errors of each experiment are within the range of $1 \%$ from Fig. 11, so the estimation precision of parameters is very high.

\subsection{Experimental results}

Experiments based on a $0.33 \mathrm{THz}$ radar system are carried out. The terahertz radar system adopted in this paper is based on linear frequency modulated (LFM) pulse principle and has a $322 \mathrm{GHz}$ of central frequency with a synthetic bandwidth of $10 \mathrm{GHz}$, thereby realizing a $1.5 \mathrm{~cm}$ theoretical range resolution. The terahertz signal is transmitted by the cone-shaped horn antenna with an azimuth beam angle $11^{\circ}$ after 36 times frequency multiplication of an X-band sweeping generator in the transmitting chain. The pulse repetition frequency (PRF) of the transmitting signal is 1000 , and the transmitting power is greater than $3 \mathrm{~mW}$.

The target in this paper is a warhead model which consists of three parts: a dome cone, a cylinder, and a frustum of a cone (as shown in Fig. 12). There are two motors in the model to implement functions of spin and coning, respectively. The Range profile sequence of the precession warhead model is shown in Fig. 13.
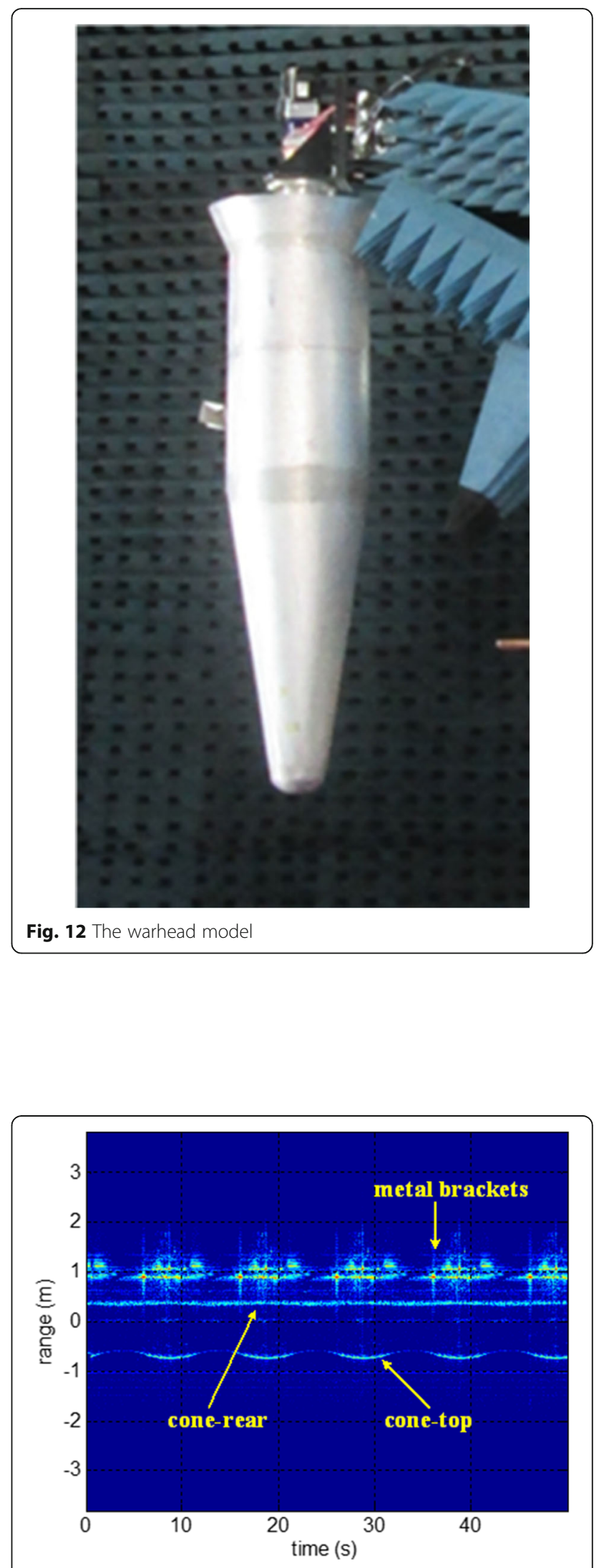

Fig. 13 The range profile sequence of the precession warhead model 

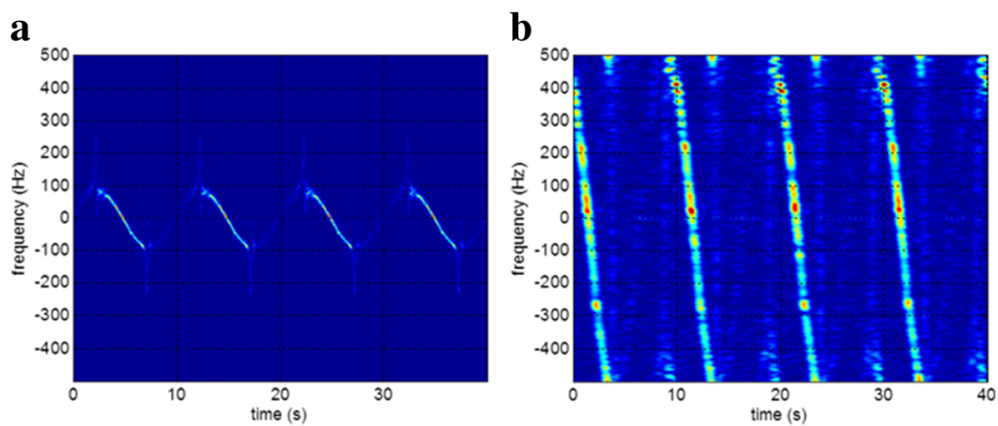

Fig. 14 Time-frequency distributions of the cone-top. a The non-aliasing situation. b The aliasing situation

We extract the range resolution bins that are related to the cone-top and analyze them by a time-frequency method to obtain the micro-Doppler curves of the cone-top. The micro-Doppler curves of cone-top at two sets of experimental parameters are shown in Fig. 14, which separately represent the two situations of aliasing and non-aliasing. The parameter spaces through the modulo GHT are shown in Fig. 15, from which we can reconfirm the excellent performance of this method on Doppler aliasing free micro-motion parameter estimation.

\subsection{Performance analysis}

\subsubsection{Computation load analysis}

The essence of our algorithm is to search the matching parameters, and the computation load is therefore closely related to the step lengths of the searching parameters. If the upper limit of the micro-Doppler frequency is $F$, and the micro-Doppler step length is $\Delta f$, the search requires $F / \Delta f$ cycles in the frequency search. Likewise, $360 / \Delta \varphi$ cycles are required in the phase search. There is only one averaging operation in each cycle. If the sampling time is $\Delta t$, and the integration time is $T$. Then the number of addition operation in a cycle is $(T / \Delta t-1+1)$, adding a division operation which is equivalent to an addition operation. The computation load $C$ is approximated as:

$$
C=\frac{F}{\Delta f} \times \frac{360}{\Delta \varphi} \times(T / \Delta t-1+1)
$$

The computation load is directly proportional to the upper limit frequency and the integration time and inversely proportional to the frequency and phase precision from Eq. (3). So, we need to make a tradeoff between the computation load and parameter estimation precision or adopt the variable step-length searching strategy mentioned above.

\subsubsection{SNR analysis}

This algorithm has a good performance in noise immunity because it has a coherent integration processing. In order to analyze the relationship between the estimation precision and signal-to-noise ratio (SNR), we plot the errors when SNR varies from -20 to $3 \mathrm{~dB}$ at $220 \mathrm{GHz}$ in Fig. 16. It is clear that the algorithm possesses good robustness and high precision when the SNR is above $-12.5 \mathrm{~dB}$, the average errors are lower than $1 \%$. When SNR is below $-12.5 \mathrm{~dB}$, the algorithm is invalid because the time-frequency distribution images are severely polluted by noise. Errors in this situation are random and there are no detectable peaks in parameter spaces. To sum up, the modulo GHT method has a good performance with SNRs above $-12.5 \mathrm{~dB}$.
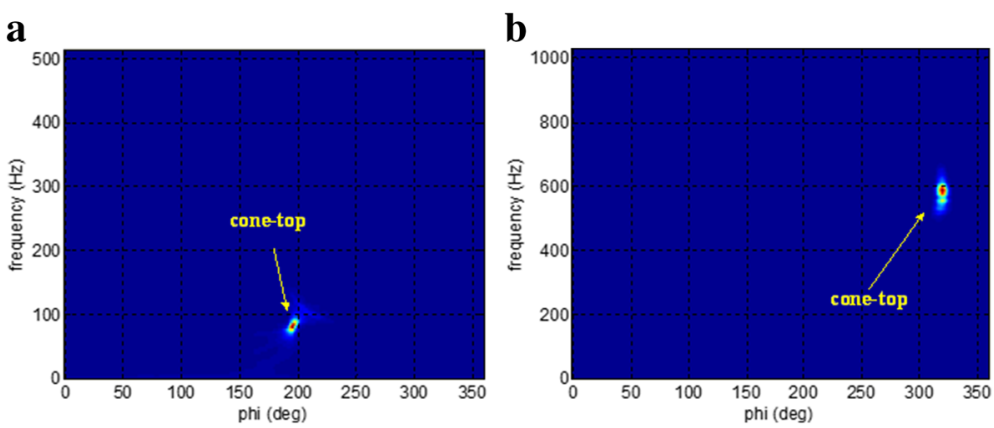

Fig. 15 Parameter spaces. a The non-aliasing situation. $\mathbf{b}$ The aliasing situation 


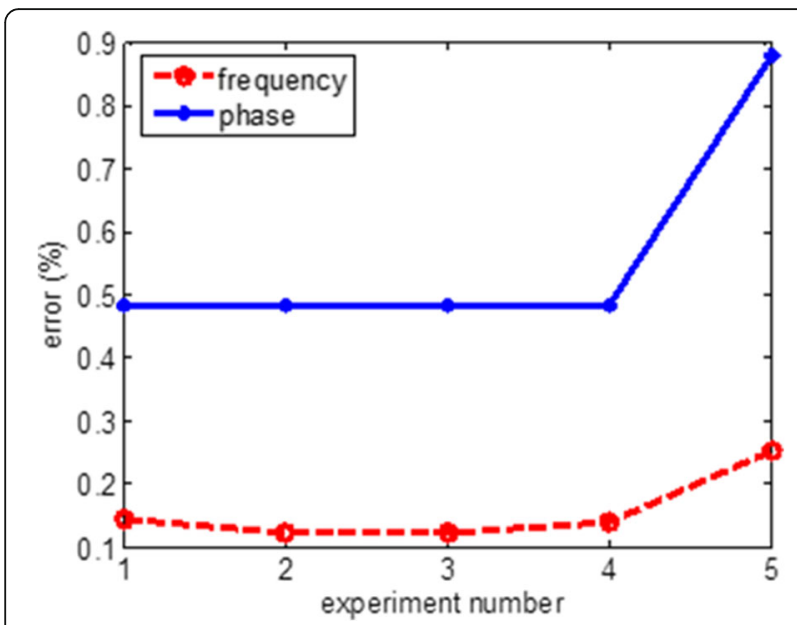

Fig. 16 Errors at different SNR

\section{Conclusions}

Researches on parameter estimation of micro-motion in the terahertz band are of great value to exploit the advantages of terahertz band and promoting the applications of terahertz radars. In this paper we have proposed a modulo GHT algorithm, and applied it to the estimation of micro-motion parameters in micro-Doppler aliasing situation. Its essence is to search the matching parameters of micro-Doppler curves in time-frequency distribution images, and it is especially suited for the micro-Doppler aliasing situation in the terahertz band. The simulation and experimental results demonstrate the high estimation precision and excellent noise immunity, especially when the SNR is above $-12.5 \mathrm{~dB}$.

\section{Acknowledgements}

The authors would like to thank the editors and reviewers for their insightful comments.

\section{Funding}

Not applicable.

\section{Authors' Contributions}

The problem of Doppler aliasing resolution with terahertz radar was arisen from the discussions between BD and HW. YQ built the $0.33 \mathrm{THz}$ radar system and wrote Section 1. The Doppler aliasing free micro-motion parameter estimation method was derived and implemented by QY. HW wrote the Abstract and BD wrote the Conclusions. Section 2, Section 3 and Section 4 were written by QY. All authors read and approved the final manuscript.

\section{Competing interests}

The authors declare that they have no competing interests.

\section{Consent for publication}

Not applicable.

Ethics approval and consent to participate Not applicable.

\section{Publisher's Note}

Springer Nature remains neutral with regard to jurisdictional claims in published maps and institutional affiliations.
Received: 27 September 2016 Accepted: 21 March 2017

Published online: 31 March 2017

\section{References}

1. I Hosako, N Sekine, M Patrashin et al., At the dawn of a new era in terahertz technology. Proc. IEEE 95(8), 1611-1623 (2007). doi:10.1109/JPROC.2007.898844

2. JF Federici, B Schulkin, F Huang et al., THz imaging and sensing for security applications-explosives, weapons and drugs. Semicond. Sci. Technol. 20(7), 266-280 (2005). doi:10.1088/0268-1242/20/7/018

3. AG Davies, AD Burnett, W Fan et al., Terahertz spectroscopy of explosives and drugs. Mater. Today 11(3), 18-26 (2008). doi:10.1016/S1369-70

4. RJ Dengler, KB Cooper, G Chattopadhyay et al., $600 \mathrm{GHz}$ imaging radar with 2 $\mathrm{cm}$ range resolution (2007 IEEE/MTT-S International Microwave Symposium, Honolulu, 2007), pp. 1371-1374. doi:10.1109/MWSYM.2007.380468

5. T Bryllert, KB Cooper, RJ Dengler et al., A $600 \mathrm{GHz}$ imaging radar for concealed objects detection (2009 IEEE Radar Conference, Pasadena, 2009), pp. 1-3. doi:10.1109/RADAR.2009.4977038

6. H Essen, A Wahlen, R Sommer et al., Development of a 220-GHz experimental radar (2008 German Microwave Conference, Hamburg-Harburg, 2009), pp. 1-4

7. KB Cooper, RJ Dengler, G Chattopadhyay et al., A high-resolution imaging radar at $580 \mathrm{GHz}$. IEEE Microwave Wireless Compon. Lett. 18(1), 64-66 (2008). doi:10.1109/LMWC.2007.912049

8. MA Basten, JC Tucek, DA Gallagher et al., A $0.85 \mathrm{THz}$ vacuum-based power amplifier (13th International Vacuum Electronics Conference, Monterey, 2012), pp. 39-40. doi:10.1109/IVEC.2012.6262066

9. M Caris, S Stanko, S Palm et al., $300 \mathrm{GHz}$ radar for high resolution SAR and ISAR applications (2015 16th International Radar Symposium, Dresden, 2015), pp. 577-580. doi:10.1109//RS.2015.7226313

10. RW Mcmillan Jr, CW Trussell, RA Bohlander et al., An experimental $225 \mathrm{GHz}$ pulsed coherent radar. IEEE Trans. Microwave Theory Tech. 39(3), 555-562 (1991). doi:10.1109/22.75300

11. J Li, Y Pi, X Yang, Micro-doppler signature feature analysis in terahertz band. J. Infrared Millimeter Terahertz Waves 31(3), 319-328 (2010). doi:10.1007/ s10762-009-9589-0

12. $Z \mathrm{ZXu}, J \mathrm{Tu}, \mathrm{J}$ Li et al., Research on micro-feature extraction algorithm of target based on terahertz radar. EURASIP J. Wirel. Commun. Netw. 2013(1), 1-9 (2013). doi:10.1186/1678-1499-2013-77

13. Y Qi, D Bin, W Hongqiang et al., Doppler aliasing free micro-motion parameter estimation algorithm based on the spliced time-frequency image and inverse Radon transform (2014 International Conference on Information and Communications Technologies (ICT 2014), Nanjing, 2014), pp. 1-6. doi:10.1049/cp.2014.0607

14. D Pao, HF Li, R Jayakumar, Detecting parameteric curves using the straight line Hough transform (10th International Conference on Pattern Recognition, Atlantic City, 1990), pp. 620-625. doi:10.1109/ICPR.1990.118175

15. PK Ser, WC Siu, Sampling Hough algorithm for the detection of lines and curves (IEEE International Symposium on Circuits and Systems, San Diego, 1992), pp. 2497-2500. doi:10.1109/ISCAS.1992.230479

16. H Li, H Zheng, Y Wang, Segment hough transform - a novel hough-based algorithm for curve detection (Fourth International Conference on Image and Graphics (ICIG 2007), Sichuan, 2007), pp. 471-477. doi:10.1109/ICIG.2007.114

17. Z Sun, B Li, Y Lu, Research on micro-motion and micro-Doppler of ballistic targets (2009 IET International Radar Conference, Guilin, 2009), pp. 1-4. doi:10.1049/cp.2009.0189

\section{Submit your manuscript to a SpringerOpen ${ }^{\mathcal{O}}$ journal and benefit from:}

- Convenient online submission

- Rigorous peer review

- Immediate publication on acceptance

- Open access: articles freely available online

- High visibility within the field

- Retaining the copyright to your article

Submit your next manuscript at $>$ springeropen.com 